\title{
BEAM TEST OF A GRID-LESS MULTI-HARMONIC BUNCHER*
}

\author{
P.N. Ostroumov, V.N. Aseev, A. Barcikowski, B. Clifft, R. Pardo, S.I. Sharamentov, M. Sengupta, \\ ANL, Argonne, IL 60439, U.S.A.
}

\begin{abstract}
The Argonne Tandem Linear Accelerator System (ATLAS) is the first superconducting heavy-ion linac in the world. Currently ATLAS is being upgraded with the Californium Rare Ion Breeder Upgrade (CARIBU). The latter is a funded project to expand the range of shortlived, neutron-rich rare isotope beams available for nuclear physics research at ATLAS. To avoid beam losses associated with the existing gridded multi-harmonic buncher (MHB), we have developed and built a grid-less four-harmonic buncher with fundamental frequency of 12.125 MHz. In this paper, we report the results of the MHB commissioning and ATLAS beam performance with the new buncher.
\end{abstract}

\section{INTRODUCTION}

A multi-harmonic buncher (MHB) is an effective system for bunching low-intensity DC ion beams upstream of an rf linac. The MHB for the ATLAS was developed long ago [1] and has been successfully operated for several decades. This ATLAS MHB consists of four-harmonic lumped-element resonant structures loaded with the parallel grids. A recent upgrade of the four-harmonic rf electronics was presented at PAC'05 [2]. The use of two grids reduces beam transmission by about $20 \%$ which is unacceptable in the acceleration of secondary radioactive beams. With the progress in a developing radioactive ion beam source coupled to ATLAS [3], we have decided to replace the MHB grids with a grid-less bunching system.

\section{DESIGN OF THE BUNCHER}

Following the developments at TRIUMF [4], we have selected a bunching system as two drift tubes connected to opposite sign of rf voltage. The side cross-section and side view of the MHB are shown in Fig. 1. This configuration allows us to use the multi-harmonic rf electronics of the old buncher [2]. The MHB aperture diameter is $20 \mathrm{~mm}$ and the voltage gap is $8 \mathrm{~mm}$. The axial field distribution obtained from Electromagnetic Studio (EMS) [5] simulation is shown in Fig. 2.

The multi-harmonic buncher must produce a saw-tooth modulation of the beam energy as shown in Fig. 3. This energy modulation produces a short bunch width at the entrance of the first Positive Ion Injector (PII) cavity. The energy gain of a charged particle passing through the gridless MHB depends on the transit time factor which is a function of both the particle velocity and harmonic

\footnotetext{
* This work was supported by the U.S. Department of Energy, Office of Nuclear Physics, under Contract No. DE-AC-02-06CH11357.

\#ostroumov@phy.anl.gov
}

number. As an example, the optimal mixture of the harmonics to produce required energy modulation of the $35 \mathrm{keV} / \mathrm{u}{ }^{58} \mathrm{Ni}^{15+}$ ion beam for the buncher with and without grids is shown in Table 1 . The harmonic mixture of the grid-less MHB also depends on beam velocity as is shown in Table 1.

The voltage amplitudes of the harmonics in the MHB are tuned using a signal from a capacitive pick-up. To set the correct harmonics mixture, the signal from the pick-up must be tuned to obtain a signal waveform as is shown in Fig. 4. In routine ATLAS operation, the ECR is tuned to produce $\sim 35 \mathrm{keV} / \mathrm{u}$ ion beams to inject into the PII. For high $\mathrm{q} / \mathrm{A}$ beams the ECR can provide higher injection energy. The optimal tuning of the MHB should be changed if the beam energy departs from the original value by more than $\sim 10 \%$. The correct waveform of the pick-up signal can be generated using data from beam dynamics simulations in realistic 3D fields by the TRACK code [6].
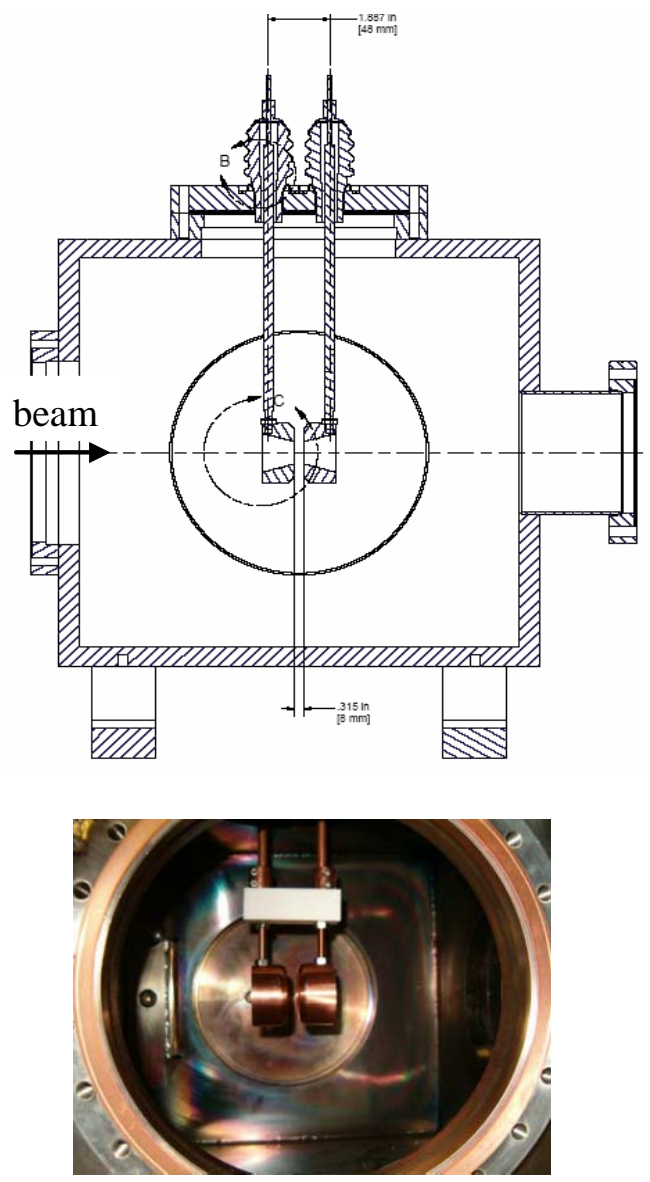

Figure 1: The side cross-section (on the top) and the side view (on the bottom) of the buncher. 

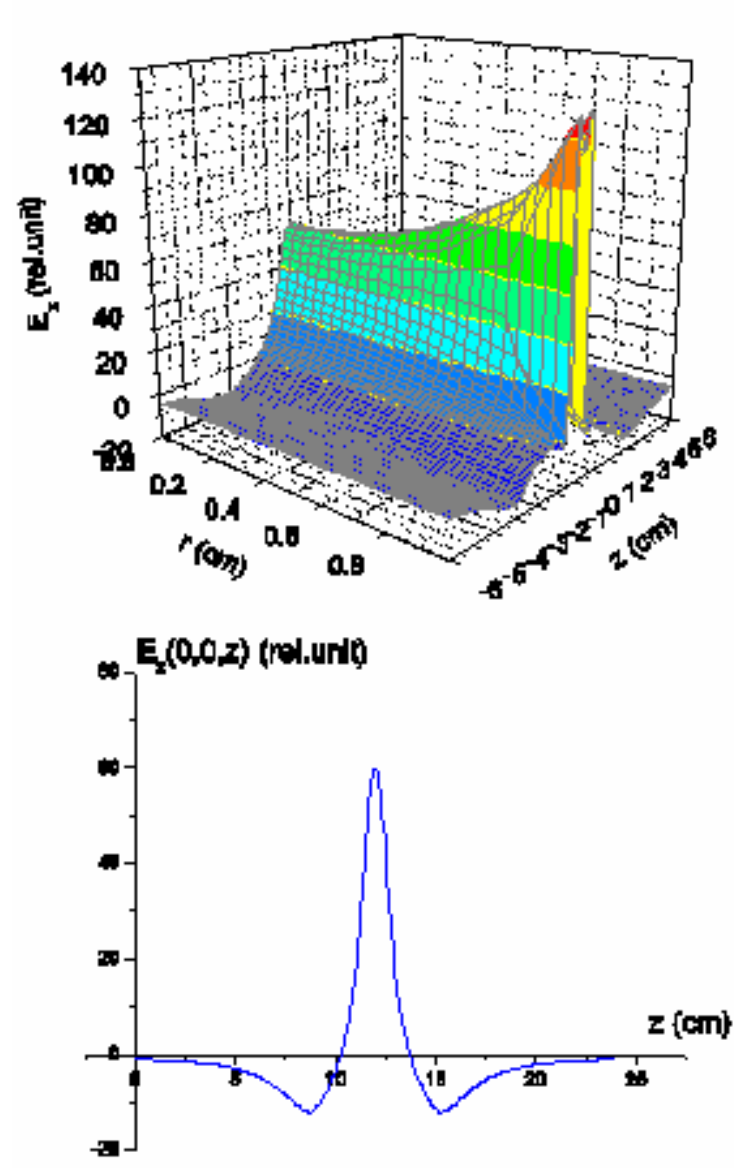

Figure 2: Electric field distribution in the MHB.

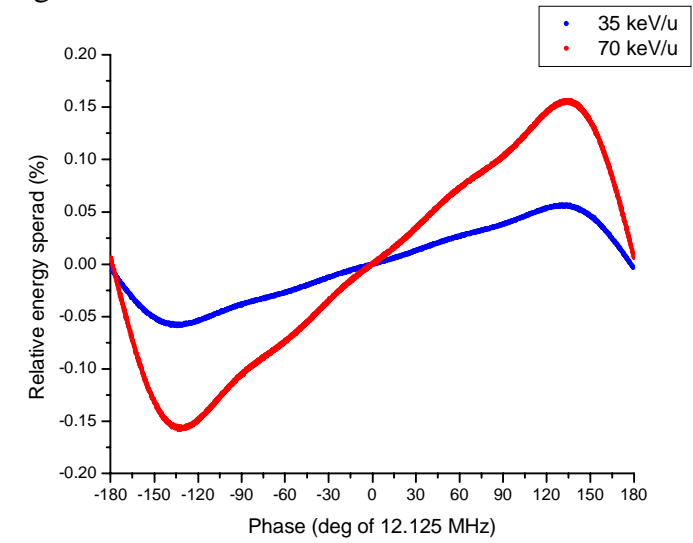

Figure 3: Relative energy modulation downstream of the buncher optimized for two different energies of ${ }^{58} \mathrm{Ni}^{15+}$ beam.

Table 1: Voltage amplitudes of different harmonics (h) calculated for the ${ }^{58} \mathrm{Ni}^{15+}$ ion beam.

\begin{tabular}{|l|l|l|l|}
\hline $\mathbf{h}$ & $\begin{array}{l}\text { With grids } \\
\mathrm{W}_{\text {in }}=35 \mathrm{keV} / \mathrm{u}\end{array}$ & $\mathrm{W}_{\text {in }}=35 \mathrm{keV} / \mathrm{u}$ & $\mathrm{W}_{\text {in }}=70 \mathrm{keV} / \mathrm{u}$ \\
\hline 1 & 2214 & 2304 & 10200 \\
\hline 2 & -891.8 & -579.8 & -1822 \\
\hline 3 & 401.4 & 267.1 & 715.7 \\
\hline 4 & -153.1 & -139.4 & -278.0 \\
\hline
\end{tabular}


the bunch width was measured to be 0.7 ns FWHM, as seen in Fig 6, in good agreement with TRACK predictions as is seen from Fig. 5. The space charge effects on bunching in this configuration can be seen in Table 2 which shows the bunch width systematically increasing to 1.2 ns for a beam current of over $50 \mu \mathrm{A}$. Similar results have been observed when the buncher was operated at single fundamental harmonic as is seen from Table 3 .

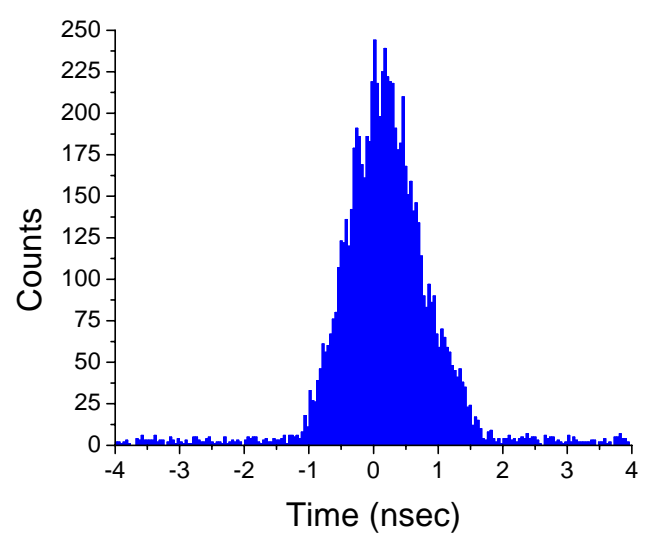

Figure 5: Bunch intensity distribution at the PII entrance.

The harmonic buncher operates in conjunction with a 24.25 MHz spiral loaded 2-gap resonator which rebunches the beam to reduce the bunch width into the first resonator further and to reduce the sensitivity to space charge. Nominally the additional 'magnification' from this buncher was expected to be approximately 0.3 . The two bunchers can operate such that the second buncher is presented with either a 'virtual' or a 'real' waist from the harmonic buncher. TRACK calculations predicted that the virtual waist would be the better operating mode.

Table 2: Space-charge effect on time width of beam waist observed for ${ }^{40} \mathrm{Ar}^{8,9+}$ beam with harmonic buncher.

\begin{tabular}{|c|c|l|}
\hline $\begin{array}{c}\text { Beam Current } \\
(\mu \mathrm{A})\end{array}$ & $\begin{array}{l}\text { Measured } \\
\text { FWHM (ns) }\end{array}$ & $\begin{array}{l}\text { Required } \\
\text { Buncher } \\
\text { Relative Amp. }\end{array}$ \\
\hline 0.3 & 0.7 & 1.0 \\
\hline 10.0 & 1.2 & 1.0 \\
\hline 25.0 & 1.2 & 1.07 \\
\hline 50.0 & 1.22 & 1.12 \\
\hline
\end{tabular}

Table 3: Comparison of the measured and simulated (in the brackets) FWHM and relative buncher amplitudes for single-harmonic buncher for ${ }^{40} \mathrm{Ar}^{8+}$.

\begin{tabular}{|c|l|l|}
\hline $\mathrm{I}(\mu \mathrm{A})$ & FWHM (ns) & $\mathrm{V}_{\mathrm{B}} / \mathrm{V}_{\mathrm{B} 0}$ \\
\hline 4.0 & $1.0(0.75)$ & $1.0(1.0)$ \\
\hline 10.0 & $1.1(0.8)$ & $1.0(1.0)$ \\
\hline 50.0 & $1.4(1.2)$ & $1.22(1.11)$ \\
\hline 85.0 & $2.0(1.5)$ & $1.22(1.16)$ \\
\hline
\end{tabular}

This was tested using a ${ }^{58} \mathrm{Ni}^{15+}$ beam at low $(\sim 1.5 \mu \mathrm{A})$ beam current. The transmission through the first section of the linac (18 resonators accelerating the beam to approximately $1.5 \mathrm{MeV} / \mathrm{u}$ ) was observed for both cases. For the virtual waist condition the best transmission achieved is $73 \%$ of the original DC beam and only $68 \%$ for the real waist configuration. TRACK calculations predicted $75 \%$ and $65 \%$ for the two situations respectively. The TRACK calculations indicate that the limitation in the real waist condition is the use of only four harmonics and the nonlinearities induced by those harmonics which come to dominate over other effects when large amplitudes are required.

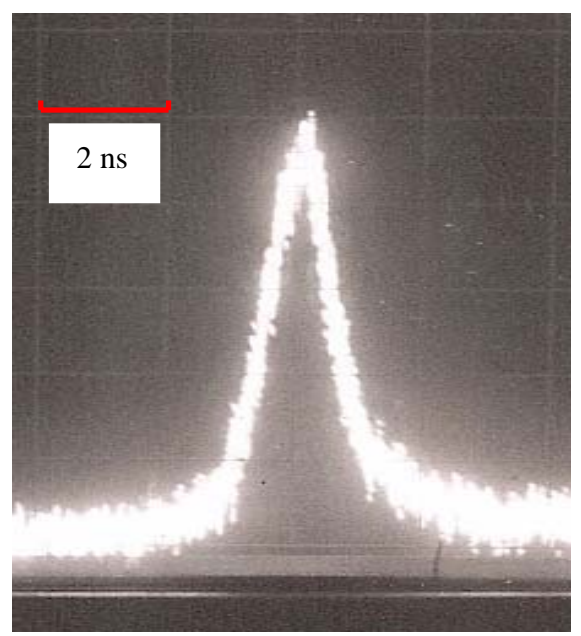

Figure 6: ${ }^{40} \mathrm{Ar}^{9+}$ bunched beam with harmonic buncher only. Beam current is $0.3 \mu \mathrm{A}$. Bunch width is $0.7 \mathrm{~ns}$ FWHM.

\section{REFERENCES}

[1] F.L Lynch, et al. Nucl. Instr, and Meth. 120 (1974), 245.

[2] S. Sharamentov, J. Bogaty, B.E. Clifft, R. Pardo, PAC’05, Knoxville, TN, May 2005, p. 2161.

[3] R. C. Pardo, G. Savard, S. Baker, C. Davids, E. F. Moore, R. C. Vondrasek, G. Zinkann,”Radioactive beams from ${ }^{252} \mathrm{Cf}$ fission using a gas catcher and an ECR charge breeder at ATLAS”, Nucl. Inst. \& Meth., to be published.

[4] L. Root, TRIUMF Design Note, Vancouver, Canada, 1998.

[5] CST Microwave Studio, http://www.cst.com/.

[6] B. Mustapha, V.N. Aseev, E. S. Lessner and P.N. Ostroumov, USA., May 2005, p. 2053.

[7] J.M. Bogaty, R.C. Pardo and B.E. Clifft, LINAC'90, Albuquerque, NM, September 1990, LA-12004-C, p. 465. 